\title{
Synapsis and recombination in intra- and interspecies hybrids between two voles species Microtus (Alexandromys) evoronensis and M. maximowizcii
}

\author{
T. Bikchurina ${ }^{1,2 *}$, T. Vasil'eva ${ }^{3}$, M. Pavlenko ${ }^{3}$, I. Sheremet'eva ${ }^{3}$, I. Kartavtseva $^{3}$ \\ ${ }^{1}$ Institute of Cytology and Genetics SB RAS, Novosibirsk, Russia \\ ${ }^{2}$ Novosibirsk State University, Novosibirsk, Russia \\ ${ }^{3}$ Federal Scientific Center of the East Asia Terrestrial Biodiversity FEB RAS, Vladivostok, Russia \\ *e-mail: bikchurina@bionet.nsc.ru
}

Key words: chromosomal rearrangements, pachytene, recombination, voles

Motivation and Aim: In the early stages of speciation, reproductive isolation can occur due to genetic incompatibility and/or gradual accumulation of different chromosomal rearrangements. Heterozygotes for the chromosomal rearrangements form complex multivalents in meiotic prophase I, which result in high occurrence of non-homologous synapsis and asynapsis of homologous chromosomes. These aberrations result in apoptosis of the hybrid gametocytes. However, cytological basis of these processes remains poorly understood. Intra- and interspecies hybrids between parental forms differed by chromosomal rearrangements provide a good model to analyze different degree of meiotic abnormalities at the early stages of speciation.

Methods and Algorithms: Using immunolocalization of SYCP3 (the main protein of lateral element of synaptonemal complex), MLH1 (miss-match protein marking late recombination nodules) and centromere proteins at pachytene we analyzed synapsis and recombination in female and male hybrids between different chromosomal races of M. evoronensis and between M. evoronensis and closely related M. maximowizcii.

Results: We found a significant difference in the degree of meiotic abnormalities between female and male interspecies hybrids. In females, we observed simple multivalents with recombination at the homologously paired arms. In males, most cells contained complex multivalents with completely suppressed recombination. Intraspecies hybrids showed different level of multivalent complexity. The number of recombination event per cell was slightly reduced compared to the parental species.

Conclusion: The severity of meiotic abnormalities in analyzed hybrid voles increased with increasing degree of chromosomal divergency. In the interspecies hybrids, the complexity of multivalents was higher and the recombination was affected stronger compared to intraspecies hybrids. In accordance to the Haldane rule, the meiotic disturbances were more pronounced in the heterogametic sex.

Acknowledgements: Supported by the by Russian Foundation for Basic Research (Grants No. 17-29-08019 and No. 15-04-03871) and the Federal Agency for Scientific Organizations via the Institute of Cytology and Genetics (Grant No. 0324-2018-0019). 